\title{
Structure of Nascent Microbial Cellulose VI. Influence of Positions of Sulfonate Groups in Fluorescent Brightener on Crystal Structure of Microbial Cellulose
}

\author{
Akira $\mathrm{KAI}^{\dagger}$ and Sherif M. A. S. KeSHK \\ Department of Applied Chemistry, Tokyo Metropolitan University, \\ Minami-Ohsawa, Hachioji, Tokyo 192-0397, Japan
}

(Received July 7, 1998)

\begin{abstract}
The influence of the number of sulfonate groups in a Fluorescent Brightener with 2 (FB) or 4 sulfonate groups (FWA1 with 2 sulfonate groups at the meta position of the phenylamino ring and FWA2 with 2 groups at the para position) and positions on the crystal structure of microbial cellulose was examined by X-ray diffraction and solid state ${ }^{13} \mathrm{C}$ NMR spectroscopy. All products obtained from Acetobacter xylinum culture in the presence of each Fluorescent Brightener were crystalline complexes containing a dye between cellulose sheets corresponding to the (110) planes of microbial cellulose. Celluloses regenerated from FB or FWA1-cellulose complexes formed cellulose $\mathrm{I}_{\beta}$ rich structures, whereas cellulose regenerated from FWA2-cellulose complex formed cellulose II. Even if a Fluorescent Brightener had the same number of sulfonate groups, the effects on the crystal structure of regenerated microbial cellulose clearly differed according to position.

KEY WORDS Acetobacter xylinum / Fluorescent Brightener / Sulfonate Group / Dye-Cellulose Complex / Cellulose $\mathrm{I}_{\beta}$ / Cellulose II /
\end{abstract}

Although Acetobacter xylinum (A. xylinum), a cellulose producing bacterium, ordinarily produces cellulose $\mathrm{I}_{\alpha}$ rich fibrils outside the cell, ${ }^{1}$ cellulose produced in the presence of a Fluorescent Brightener (FB) does not form such fibrils but forms bands. ${ }^{2,3}$ This product is a crystalline complex containing a dye molecule layer between cellulose sheets corresponding to the (110) plane of microbial cellulose..$^{4-7}$ When the complex is dye-extracted, cellulose, which is not the structure of ordinary microbial cellulose but a cellulose $\mathrm{I}_{\beta}$ rich structure, ${ }^{6}$ is regenerated from the complex. A complex with a similar structure is produced from A. xylinum culture in the presence of Direct Red 28, but cellulose $\mathrm{IV}_{\mathrm{I}}$ is regenerated. ${ }^{8}$ Celluloses, regenerated from complexes produced in the presence of Direct Blue 1, 14, 15, or 53 having the same skeletal structure as Direct Red 28 and 2 sulfonate groups more than Direct Red 28, form cellulose II. ${ }^{9,10}$ Although the influence on the crystal type of cellulose regenerated from some complexes likely depends on interactions between a dye and cellulose, the number of sulfonate groups per unit of molecular weight of a dye seems predominant. ${ }^{11}$

In this study, the influence of the number of sulfonate groups in the FB was assessed. Two other sulfonate groups at the meta (FWA1) or para (FWA2) position of the phenylamino ring, and position on the crystal structure of microbial cellulose were examined by X-ray diffraction and solid state ${ }^{13} \mathrm{C}$ NMR spectroscopies.

\section{EXPERIMENTAL}

\section{Preparation of Samples}

Chemical Structures of Direct Dyes. The direct dyes used in this experiment are FB (4,4'-bis-[4-anilino-6bis(2-hydroxylethyl)amino-1,3,5-triazin-2-ylamino]-2,2'stilbene disulfonate, Nippon Kayaku), FWA1 (tetra-

\footnotetext{
$\dagger^{\dagger}$ To whom correspondence should be addressed
}

sodium 4,4'-bis[4-bis(2-hydroxyethyl)amino-6-(3-sulfonatephenylamino)-1,3,5-triazin-2-ylamino]stilbene2,2'-disulfonate, Nippon Kayaku), and FWA2 (tetrasodium 4,4'-bis[4-bis(2-hydroxyethyl)amino-6-(4-sulfonatophenylamino)-1,3,5-triazin-2- ylamino]stilbene-2,2' disulfonate, Nippon Kayaku). The chemical structures of these dyes are shown in Figure 1.

Dye-Cellulose Complex. A. xylinum (IFO 13693) was cultured in a $15 \mathrm{~cm}$ diameter petridish containing $110 \mathrm{ml}$ Hestrin-Schramm (HS) medium ${ }^{12}\left(\mathrm{pH} \mathrm{6.8)}\right.$ at $28^{\circ} \mathrm{C}$ for 3 days in an incubator. $60 \mathrm{ml}$ of cellulose-free cell suspension, prepared from matured petridish in $120 \mathrm{ml}$ phosphate buffer solution, were added to $140 \mathrm{ml}$ of HS medium ( $\mathrm{pH} 7.0$ ) containing a direct dye of $0.1 \mathrm{wt} \%$ and the mixture was incubated at $28^{\circ} \mathrm{C}$ for $24 \mathrm{~h}$ under static conditions. The product was collected by centrifuging $(6000 \mathrm{rpm}, 5.15 \mathrm{~g}, 20 \mathrm{~min})$. The product obtained from the medium containing FB was washed well with $0.1 \mathrm{wt} \%$

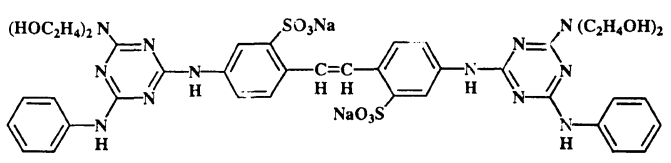

FB
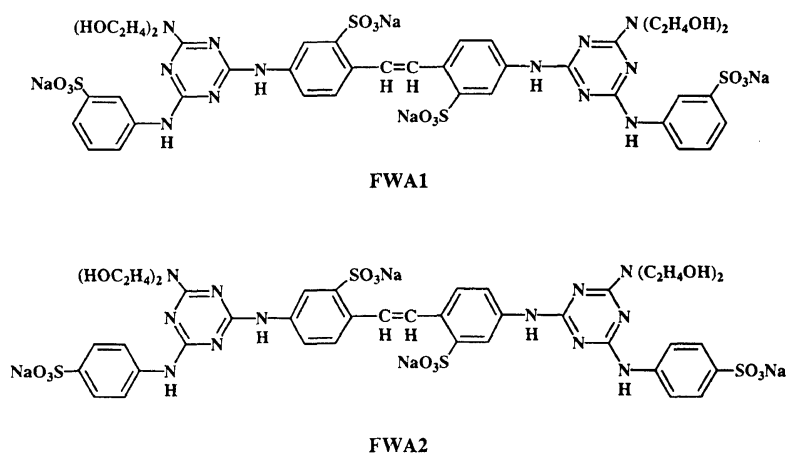

Figure 1. Chemical structures of the direct dyes used. 
$\mathrm{NaOH}$ aqueous solution to remove the dye not related to dyeing and with distilled water until alkali-free. The product obtained in the presence of FWA1 or FWA2 was rinsed only with distilled water for $60 \mathrm{~min}$. These products were preserved at about $3^{\circ} \mathrm{C}$ in the wet state.

Regenerated Cellulose Sample. Dye extraction of the above samples was performed by boiling in $70 \mathrm{vol} \%$ aqueous ethanol solutions for $18 \mathrm{~h}$ and the fresh aqueous ethanol solution was exchanged every $3 \mathrm{~h}$. This dye extracted sample was again boiled in $1.0 \mathrm{wt} \%$ aqueous sodium hydroxide solution for $10 \mathrm{~h}$ under $\mathrm{N}_{2}$ atmosphere to remove protein. The regenerated cellulose sample was neutralized with $1.0 \mathrm{vol} \%$ aqueous acetic acid and then rinsed with distilled water. The sample was preserved as above.

Control Cellulose Samples. Control cellulose I sample was prepared by washing microbial cellulose with distilled water to remove medium components and boiled in $1.0 \mathrm{wt} \%$ aqueous sodium hydroxide solution for $10 \mathrm{~h}$ under $\mathrm{N}_{2}$ atmosphere. The final sample was neutralized and rinsed as above. The cellulose II sample was prepared by mercerization of a microbial cellulose at $20^{\circ} \mathrm{C}$ for $1 \mathrm{~h}$.

\section{Methods for the Measurements}

$X$-Ray Measurement. The X-ray diffractogram of an uniplanar oriented membrane of a product was measured by reflection and transmission. The membrane was prepared from the preserved product and dried on a Teflon plate at room temperature. The diffractograms of an isotropic product and regenerated cellulose were performed by transmission. In this method, the sample was compressed as a pellet into the pore of a sample holder of $2 \mathrm{~mm}$ in diameter and $1 \mathrm{~mm}$ thickness. The $\mathrm{X}$-ray diffractogram was measured by an $\mathrm{MXP}^{18}$ diffractometer (MAC Science) with Ni-filtered $\mathrm{Cu}-K_{\alpha}$ radiation. The measurement conditions were as follows: divergence slit, $1.0 \mathrm{~mm}$; receiving slit, $0.15 \mathrm{~mm}$; scanning speed, $4.0^{\circ} \mathrm{min}^{-1}$; X-ray radiation, $40 \mathrm{kV}, 200 \mathrm{~mA}$.

Solid State ${ }^{13}$ C NMR Spectroscopy. Solid state ${ }^{13} \mathrm{C}$ NMR experiments were performed at room temperature on a JEOL JNM EX 270 spectrometer operating at 6.35 T. The MAS rate was $5.0-5.5 \mathrm{kHz}$ and chemical shifts relative to tetramethylsilane were determined by the crystalline peak at $17.3 \mathrm{ppm}$ of solid hexamethyl benzene as an external standard. The non-dried samples were packed in a MAS rotor with an O-ring seal to avoid the loss of water during NMR measurements. The spectrum of the crystalline component was measured selectively taking NMR signals after a relaxation of the noncrystalline component. To determinine the crystalline components of the control and regenerated celluloses, signals obtained after $\tau=50 \mathrm{~s}$ were measured using T1CP pulse sequence ${ }^{13}$ with CP except for microbial cellulose and cotton. The spectra of microbial cellulose and cotton were from previous paper. ${ }^{6}$

\section{RESULTS AND DISCUSSION}

Figure 2 shows the $\mathrm{X}$-ray diffractograms of isotropic samples of microbial cellulose and products (FB pro62

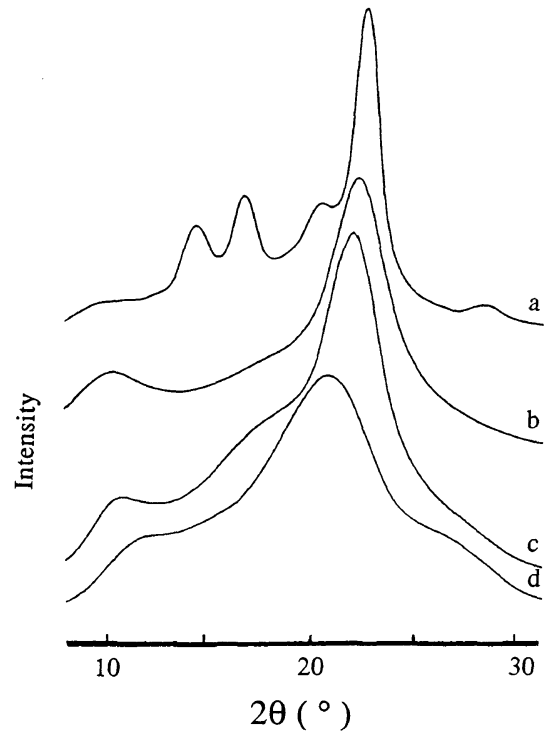

Figure 2. X-Ray diffractograms of isotropic samples of microbial cellulose and the product samples from Acetobacter culture in the presence of a dye of $0.1 \mathrm{wt} \%$. a, microbial cellulose; b, FB product; $\mathrm{c}$, FWA1 product; d, FWA2 product.

Table I. X-Ray diffraction angles of MC and products from Acetobacter-culture in the presence of FB, FWA1, or FAW 2

\begin{tabular}{llcccc}
\hline Sample & \multicolumn{5}{c}{$2 \theta / \mathrm{deg}$} \\
\hline MC & & 14.5 & 16.8 & 20.1 & 22.7 \\
FB & 10.8 & & & & 21.7 \\
FWA1 & 10.6 & & & & 22.0 \\
FAW2 & 11.0 & & & 20.7 & \\
\hline
\end{tabular}

MC, microbial cellulose.

Table II. X-Ray diffraction angles of FB, FWA1, and FWA2 products measured by transmission $(\mathrm{T})$ and reflection $(\mathrm{R})$

\begin{tabular}{lcrc}
\multirow{2}{*}{ Sample } & \multicolumn{2}{c}{$2 \theta /$ deg } \\
\cline { 3 - 4 } & & \multicolumn{2}{c}{$d$-spacing in $\AA$} \\
\hline FB product & $\mathrm{T}$ & $10.3(8.61)$ & $22.3(3.98)$ \\
& $\mathrm{R}$ & $8.9(9.88)$ & $22.1(4.02)$ \\
FWA1 product & $\mathrm{T}$ & $10.5(8.38)$ & $22.0(4.04)$ \\
& $\mathrm{R}$ & $8.3(10.7)$ & $21.6(4.10)$ \\
FWA2 product & $\mathrm{T}$ & $10.4(8.52)$ & $20.6(4.03)$ \\
& $\mathrm{R}$ & $8.7(10.1)$ & $21.2(4.18)$
\end{tabular}

duct, FWA 1 product, and FWA 2 product) obtained from A. xylinum culture in the presence of FB, FWA1, or FWA2. In Table I, $2 \theta \mathrm{s}$ of diffraction peaks in the diffractograms shown in Figure 2 are listed. Figure 3 shows X-ray diffractograms of FB, FWA1, and FWA2 product membranes measured by transmission and reflection. In Table II, $2 \theta \mathrm{s}$ of diffraction peaks from Figure 3 are listed.

As shown in Figure 2, all products from A. xylinum culture in the presence of FB, FWA1, or FWA2 show characteristic diffractograms with 2 diffraction peaks. In the diffractograms of FB and FWA1 products, 2 diffraction peaks occurred near 11 and $22^{\circ}$, whereas in the diffractogram of the FWA2 product, diffraction peaks appeared near 11 and $20.7^{\circ}$. These diffractograms were 


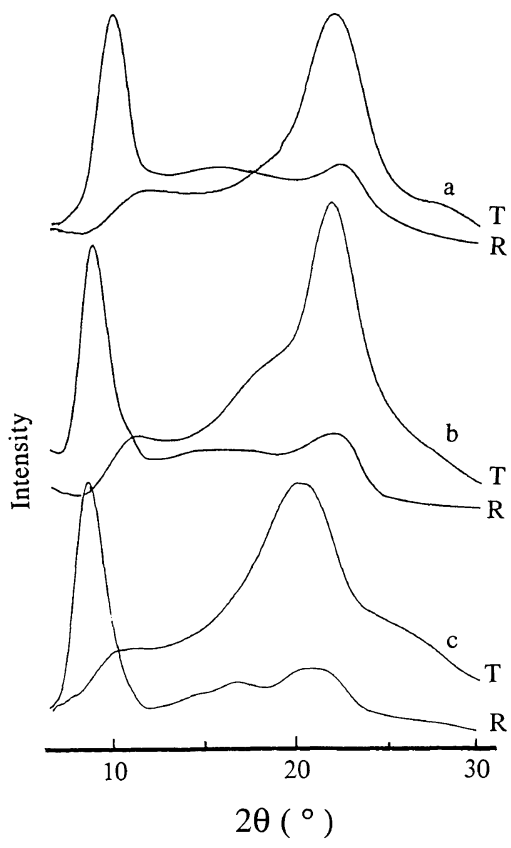

Figure 3. X-Ray diffractograms of product membranes measured by transmission (T) and reflection (R). a, FB product; b, FWAl product; c, FWA2 product.

distinctly different from that with 4 peaks of microbial cellulose. The diffractograms of products were very similar to those of products obtained from the culture in the presence of other direct dyes (Direct Red 28, 75, 79, 80, Direct Blue 1, 14, 15, 53, 71, or Direct Brown 106). ${ }^{8-11,14}$ The diffraction peak at the high angle side of the product obtained from the culture in the presence of FB, FWA 1, Direct Red $28,{ }^{8}$ Direct Blue $71,{ }^{11}$ or Direct Brown $106^{11}$ occurred at $22-23^{\circ}$, whereas that of the product in the presence of FWA 2 or others, at $20-21^{\circ}$.

As shown in Figure 3 and Table II, in the diffractograms of the uniplanar oriented membrane of the FB and FWA1 products measured by transmission with an X-ray beam transmitted perpendicular to the surface of the membrane, weak and strong peaks occurred near 10.4 and $22.0^{\circ}$, respectively. However, in the diffractogram obtained using reflection with an X-ray beam reflected to the surface of the membrane, strong and weak peaks occurred near 8.5 and $22.0^{\circ}$, respectively. In the diffractogram of the FWA2 product membrane measured by the transmission method, weak and strong peaks occurred at 10.4 and $20.6^{\circ}$, respectively, whereas, by reflection, strong and weak peaks occur at 8.7 and $21.2^{\circ}$, respectively. The diffraction planes at the low angle side of these product membranes were parallel to the surface, whereas the diffraction planes at the high angle side were perpendicular to the surface.

The diffraction plane at the low angle side likely expanded by including a dye between cellulose sheets corresponding to the $(1 \overline{1} 0)$ plane at $14.5^{\circ}$ of microbial cellulose as described previouly. The plane corresponded to the (100) plane of the complex composed of a dye and cellulose.$^{4,8,15}$ In contrast, the diffraction plane near $21^{\circ}$ likely corresponded to the (010) plane of the complex. ${ }^{4,8,15}$ The structures of FB, FWA1, and FWA2 products are similar since a dye is included between

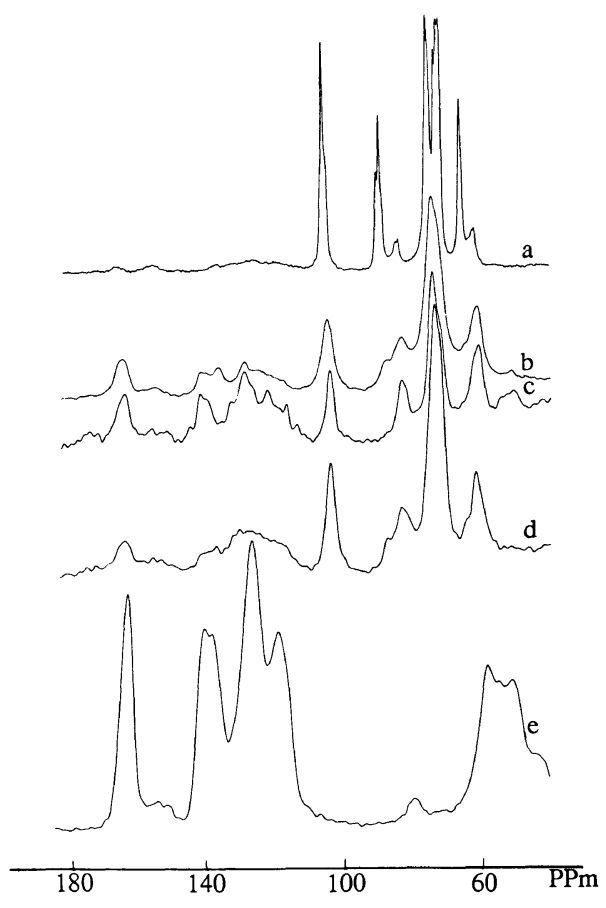

Figure 4. Solid state ${ }^{13} \mathrm{C}$ NMR spectra of wet microbial cellulose, wet products and dry direct dye. a, microbial cellulose; b, FB product; c, FWA1 product; d, FWA2 product; e, FWA2 powder.

Table III. ${ }^{13} \mathrm{C}$ chemical shifts (ppm) of microbial cellulose and products obtained from Acetobacter-culture in the presence of FB, FWA1, or FAW 2

\begin{tabular}{lcccc}
\hline Sample & C1 & C4 & C2, 3, 5 & C6 \\
\hline MC & 106.3 & $90.2,84.7$ & $75.9,73.8,72.3$ & $66.5,62.5$ \\
FB & 105.3 & -84.5 & 74.7 & -62.5 \\
FWA1 & 104.1 & -83.4 & 74.4 & -61.2 \\
FWA2 & 105.0 & -84.1 & 74.3 & -63.0 \\
\hline
\end{tabular}

MC, microbial cellulose.

cellulose sheets.

Figure 4 shows the solid state ${ }^{13} \mathrm{C}$ NMR spectra of FB, FWA1, and FWA2 complexes, FWA2 powder and microbial cellulose. The chemical shifts of resonance lines of each sample are listed in Table III. As shown in Figure 4 , no resonance lines of the cellulose component in the complex overlap the line of the dye powder. It is therefore possible to discuss the structures of cellulose components in complexes using the $\mathrm{CP} / \mathrm{MAS}{ }^{13} \mathrm{C}$ NMR spectra. The results were in agreement with our previous report. ${ }^{6}$ As shown in Figure 4 and Table III, the resonance lines of carbons of cellulose components in all complexes are broader than those of microbial cellulose. C4 and C6 resonance lines for the complexes appeared near 85.0 and $62.5 \mathrm{ppm}$, respectively, which are very close to the corresponding chemical shifts of the non-crystalline component of microbial cellulose, as shown in Table III. $\mathrm{C} 1$ resonance lines of complexes was shifted upfield by $1.0-2.2 \mathrm{ppm}$ compared to that of microbial cellulose. Although X-ray measurement indicated the complex is in a crystalline state, solid state ${ }^{13} \mathrm{C}$ NMR spectroscopy indicated the cellulose component in the complex is in a noncrystalline state. This discrepancy suggests that the mobility of the carbon of the cellulose component in the complex is almost the same as the carbon of the 


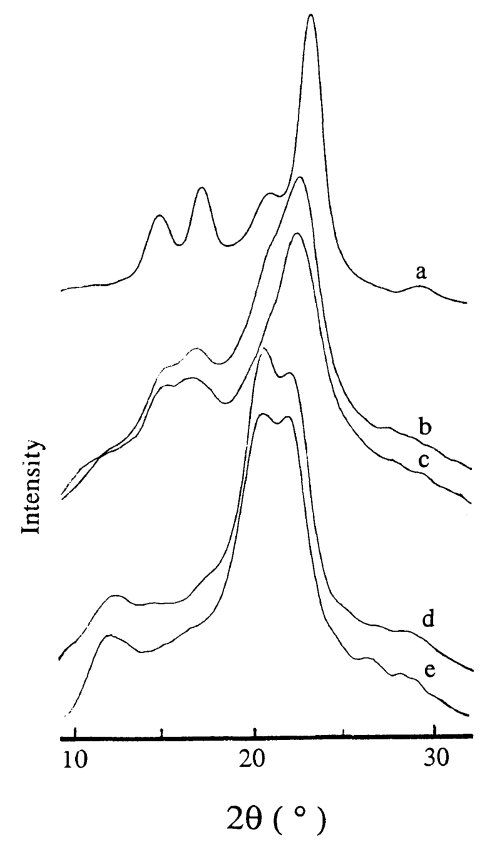

Figure 5. X-Ray diffractograms of the celluloses regenerated from the product samples. a, microbial cellulose; b, FB cellulose; c, FWA1 cellulose; d, cellulose II; e, FWA2 cellulose.

Table IV. X-Ray diffraction angles of microbial cellulose, cellulose II and celluloses regenerated from products

\begin{tabular}{lccc}
\hline \multirow{3}{*}{ Sample } & \multicolumn{3}{c}{$2 \theta / \mathrm{deg}$} \\
& $(1 \overline{1} 0)$ & $(110)$ & $(020)$ \\
\hline MC & 14.5 & 16.8 & 22.7 \\
FB & 14.4 & 16.6 & 22.3 \\
FWA1 & 14.8 & 16.3 & 22.2 \\
Cellulose II & 12.0 & 19.8 & 21.1 \\
FWA2 & 11.7 & 20.1 & 21.6 \\
\hline
\end{tabular}

noncrystalline region of microbial cellulose, because hydrogen bonding between cellulose sheets is hindered due to the dye between sheets in the complex. The spectrum of the cellulose components in each FB, FWA1, and FWA2 complex resembles each other. The structures of the complexes resemble each other since a dye is included between the cellulose sheets.

Figure 5 shows X-ray diffractograms of isotropic samples of celluloses regenerated from complexes (FB cellulose, FWA1 cellulose, and FWA2 cellulose), microbial cellulose and cellulose II. In Table IV, $2 \theta \mathrm{s}$ of the (110), (110), and (020) planes of each sample are listed.

As shown in Figure 5 and Table IV, celluloses regenerated from the FB and FWA1 complexes form cellulose I. In contrast, cellulose regenerated from the FWA2 complex forms cellulose II.

Judging from the X-ray diffraction and solid state ${ }^{13} \mathrm{C}$ NMR measurements of the complex, structures of FB, FWA1, and FWA2 complexes resemble each other. The conformation and arrangement of cellulose chains in a cellulose sheet may differ for the FB and FWA1 complex groups and FWA2 complex. In fact, in X-ray diffractograms of the complexes, $2 \theta$ of the diffraction peak at high angle differs by about $1^{\circ}$ for those of FB and FWA1 complex groups and FWA2 complex. Celluloses regenerated from complex groups with a diffrac-

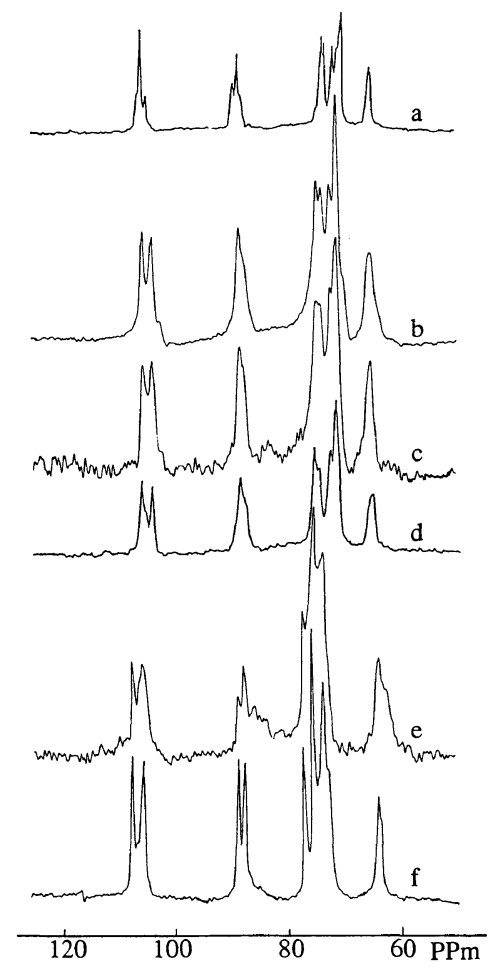

Figure 6. Solid state ${ }^{13} \mathrm{C}$ NMR spectra of crystalline components in wet cellulose regenerated from products, wet microbial cellulose, wet cotton, and cellulose II. a, microbial cellulose ${ }^{5}$; b, FB cellulose; c, FWA1 cellulose; d, cotton ${ }^{5}$; e, FWA2 cellulose; f, cellulose II.

tion peak at the high angle side at $22-23^{\circ}$, formed cellulose $I$ or $I_{1}$ while celluloses regenerated from complex groups with a diffraction peak at $20-21^{\circ}$, formed cellulose II.

The number of sulfonate groups of FWA1 and FWA2 is the same, but positions in the phenylamino ring differ. Due to hydrogen bonding between the sulfonate group at the meta position of the phenylamino ring of the FWA 1 molecule and the hydroxyethyl group in triazin ring or steric hindrance, the effect of the sulfonate group on the structure of the noncrystalline nascent microbial cellulose $^{16}$ may differ from that of the sulfonate group at the para position of the FWA2 molecule. The effects of sulfonate groups in a dye on the structure of the nascent cellulose seem to be different according to their number. The desorption of a dye from the complex during dye-extraction may also affect crystal formation of regenerated cellulose.

Solid state ${ }^{13} \mathrm{C}$ NMR spectra of the crystalline component of regenerated cellulose, microbial cellulose, cotton and cellulose II are shown in Figure 6.

As shown in Figure 6, the spectra of FB and FWA1 celluloses do not resemble that of microbial cellulose with the cellulose $I_{\alpha}$ rich structure but that of cotton with the cellulose $\mathrm{I}_{\beta}$ rich structure. In contrast, the spectrum of FWA2 cellulose distinctly shows that of cellulose II. The effects of FWA1 with 4 sulfonate groups on the crystal structure of regenerated microbial cellulose were the same as that of FB with 2 sulfonate groups, but differed from that of FWA2 with 4 sulfonate groups.

As in our previous work, ${ }^{11}$ for dye molecular weight more than 300 per sulfonate group $(M W / N, M W$, dye 
molecular weight; $N$, number of sulfonate groups), cellulose I or $\mathrm{IV}_{\mathrm{I}}$ was regenerated from the dye-cellulose complex, whereas, for $M W / N$ less than 300 , cellulose II was regenerated. ${ }^{11} \mathrm{MW} / \mathrm{N}$ of $\mathrm{FB}$ is 458 and those of FWA1 and FWA2 are 262. Nevertheless, the effect of FWA1 on the crystal structure of regenerated microbial cellulose was similar to that of FB. The different effects of FWA1 and FWA2 may be due to hydrogen bonding between the sulfonate group at the meta position of the phenylamino ring of the FWA1 molecule and hydroxyethyl group in its triazin ring or the steric hindrance as described previously. In this case, the valid number of sulfonate groups is 2 and its $M W / N$ is 523 .

Although $M W / N$ of Direct Blue 71 with 4 sulfonate

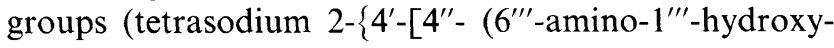
$3^{\prime \prime \prime}$-sulfonato- $2^{\prime \prime \prime}$-naphthylazo)- $6^{\prime \prime}$-sulfonato- $1^{\prime \prime}$-naphthlazo]-1'-naphthylazo $\}$ naphthalene-4,8-disulfonate) is 235, cellulose I is regenerated from the Direct Blue 71-cellulose complex. ${ }^{11}$ Due to hydrogen bonding between the sulfonate group in the $2^{\prime \prime}$-naphthylazo ring and the sulfonate group in the $1^{\prime \prime}$-naphthylazo or the steric hinderance between them, the effects of the sulfonate group in Direct Blue 71 on the crystal structure of regenerated cellulose may be similar to those of FWA1. If this is true, the valid number of sulfonate groups in Direct Blue 71 would be 3 and $M W / N, 314$.

With a $M W / N$ of more than 300 , the affinity of a dye toward cellulose increases and may protect the structure of the cellulose sheet, especially, the conformation of the cellulose chain. However, cellulose regenerated from the FB or FWA1 complex does not form the cellulose $I_{\alpha}$ rich structure of ordinary microbial cellulose but the cellulose $\mathrm{I}_{\beta}$ rich structure. The cellulose $\mathrm{I}_{\alpha}$ rich structure is considered to be formed when cellulose is crystallized under shear stress in the molecular axial direction. ${ }^{17}$ As cellulose produced in the presence of a dye relaxes through the adsorption and desorption processes of a dye toward cellulose, cellulose $\mathrm{I}_{\beta}$, which is thermodynamically more stable than cellulose $\mathrm{I}_{\alpha}$, may be formed. In contrast, FWA2, which is highly water-solube with low affinity toward cellulose, may disrupt the conformation and arrangement of cellulose chains when it adheres to nascent noncrystalline cellulose.

\section{CONCLUSIONS}

The products from $A$. xylinum culture in the presence of FB with 2 sulfonate groups, FWA1 or FWA2 with 4 sulfonate groups are crystalline complexes each containing a dye between the $(1 \overline{1} 0)$ planes of microbial cellulose. Cellulose $\mathrm{I}_{\beta}$ rich cellulose is regenerated from $\mathrm{FB}$ (with 2 sulfonate groups) or FWA1 (with 4 sulfonate groups but 2 sulfonate groups at the meta positon of the phenylamino ring)-cellulose complex, whereas cellulose II is regenerated from the FWA2 (with 4 sulfonate groups but 2 sulfonate groups at the para position of the phenylamino ring)-cellulose complex. The effects of Fluorescent Brightener with the same number of sulfonate groups on the crystal structure of regenerated microbial cellulose clearly differ according to position.

Acknowledgment. The authors are grateful to Nippon Kayaku Co., Ltd. for gifts of Fluorescent Brighteners (FB, FWA1, and FWA2).

\section{REFERENCES}

1. F. Horii, A. Hirai, and R. Kitamaru, Macromolecules, 20, 2117 (1987).

2. C. H. Haigler, R. M. Brown, Jr., and M. Benziman, Science, 210 , 903 (1980).

3. M. Benziman, C. H. Haigler, R. M. Brown, Jr., A. R. White, and K. M. Cooper, Proc. Natl. Acad. Sci. U.S.A., 77, 6678 (1980).

4. A. Kai and P. Xu, Koubunshi Ronbunshu, 48, 449 (1991).

5. A. Kai and P. Xu, Koubunshi Ronbunshu, 48, 419 (1991).

6. A. Kai, P. Xu, F. Horii, and S. Hu, Polym., 35, 75 (1994).

7. A. Kai and S. M. A. S. Keshk, Polym. J., 30, 996 (1998).

8. A. Kai and Md. I. H. Mondal, Int. J. Biol. Macromol., 20, 221 (1997).

9. Md. I. H. Mondal and A. Kai, Polym. J., 30, 78 (1998).

10. Md. I. H. Mondal and A. Kai, Polym. J., 30, 84 (1998).

11. H. Kido and A. Kai, Polym. J., 30, 508 (1998).

12. S. Hestrin and M. Schramm, Biochem. J., 58, 345 (1954).

13. D. A. Torchia, J. Magn. Reson., 30, 613 (1978).

14. A. Kai and Md. I. H. Mondal, Polym. J., 30, 398 (1998).

15. A. Kai, P. Xu, N. Ishida, and S. Ishikita, "Cellulose Sources and Exploitation," J. F. Kennedy, G. O. Phillips, and P. A. Williams, Ed., Ellis Horwood, Chichester, 1990, p 33.

16. A. Kai and T. Koseki, Makromol. Chem., 186, 2609 (1985).

17. H. Yamamoto, F. Horii, and A. Hirai, Cellulose, 3, 229 (1996). 